\title{
Intercomparisons of HIRDLS, COSMIC and SABER for the detection of stratospheric gravity waves
}

\author{
C. J. Wright ${ }^{1}$, M. B. Rivas ${ }^{1}$, and J. C. Gille ${ }^{1,2}$ \\ ${ }^{1}$ National Center for Atmospheric Research, Boulder, Colorado, USA \\ ${ }^{2}$ Center for Limb Atmospheric Sounding, University of Colorado, Boulder, Colorado, USA \\ Received: 21 December 2010 - Published in Atmos. Meas. Tech. Discuss.: 25 January 2011 \\ Revised: 10 August 2011 - Accepted: 11 August 2011 - Published: 17 August 2011
}

\begin{abstract}
Colocated temperature profiles from the Constellation Observing System for Meteorology, Ionosphere and Climate (COSMIC), High Resolution Dynamics Limb Sounder (HIRDLS) and the Sounding of the Atmosphere using Broadband Emission Radiometry (SABER) mission are compared over the years 2006-2007 to assess their relative performances for the detection of stratospheric gravity waves. Two methods are used, one based on a simple comparison of the standard deviations and correlation coefficients of high-pass filtered profiles from each instrument, and the other based on Stockwell transform analyses of the profiles for vertical wavelength and temperature perturbation scales. It is concluded, when allowing for their different vertical resolution capabilites, that the three instruments reproduce each other's results for magnitude and vertical scale of perturbations to within their resolution limits in approximately $50 \%$ of cases, but with a positive frequency and temperature bias in the case of COSMIC. This is possibly indicative of a slightly higher vertical resolution being available to the constellation than estimated.
\end{abstract}

\section{Introduction}

Atmospheric gravity waves contribute significantly to the behaviour of the atmosphere, making up an essential part of the dynamics of the atmosphere on all meteorological scales (Nappo, 2002). They strongly affect the circulation of the middle atmosphere by transporting and redistributing momentum, and are believed to largely determine the large-scale

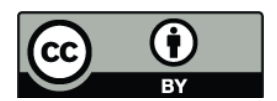

Correspondence to: C. J. Wright (cwright@ucar.edu) structure of the middle atmosphere (Fritts, 1984), accounting for the majority of temperature and velocity variances at small- and mesoscales (Fritts and Luo, 1992). Verticallypropagating gravity waves carry a flux of horizontal momentum, transferring it away from low altitudes and returning it to the mean flow at altitudes and locations far removed from the region of wave generation, via processes such as dissipation, saturation, wave-breaking and critical-level absorption (Lane et al., 2001). Inclusion of parameterisations of these processes into numerical weather prediction and climate models have significantly improved their predictive capability (Karoly et al., 1996). Accordingly, an improved understanding of gravity wave processes is important to our understanding of the climate system as a whole.

\section{Instruments}

\subsection{COSMIC}

The Constellation Observing System for Meteorology, Ionosphere and Climate (COSMIC), a joint US/Taiwanese mission, is based on a constellation of six identical microsatellites at an orbital altitude of $800 \mathrm{~km}$ in circular orbits with a $72^{\circ}$ inclination. Each satellite carries a radio occultation receiver, which is used to intercept GPS signals transmitted through the atmosphere. The phase delay in these signals allows the bending angle of the signal path through the atmosphere to be computed, which can then be analysed to produce profiles of stratospheric dry temperature (Anthes et al., 2008; Barnett et al., 2008).

COSMIC measurements require an optical path between a GPS and a COSMIC satellite, and hence the profiles are distributed pseudo-randomly across the globe. Around

Published by Copernicus Publications on behalf of the European Geosciences Union. 
1000-2500 profiles are measured globally on any given day, with temperature soundings typically covering the $5-40 \mathrm{~km}$ range (Anthes et al., 2008). Vertical resolution estimates, based on the size of the signal Fresnel zone in typical atmospheric conditions, range from $\sim 1 \mathrm{~km}$ in the stratosphere to $\sim 100 \mathrm{~m}$ in the lower troposphere, with a precision of $\sim 0.5 \mathrm{~K}$ (Anthes et al., 2008). Figure 3 of Alexander and Barnet (2007) illustrates this footprint for a typical modelled convective gravity wave. This study uses dry temperature profiles, COSMIC version 2007.3200, from the beginning of data availability in mid-2006 until the end of 2007.

\subsection{HIRDLS}

The High Resolution Dynamics Limb Sounder (Gille et al., 2003,2008 ) is a 21-channel limb-scanning infrared radiometer aboard NASA's Aura satellite, designed to measure atmospheric radiances globally at a high vertical resolution $\sim 1 \mathrm{~km}$. Aura is in a sun-synchronous low polar orbit with a period of $99 \mathrm{~min}$ and is part of the A-Train satellite constellation.

HIRDLS suffered a partial failure during launch, which further tests concluded was due to a piece of Kapton insulating material which came loose during launch covering part of the viewing window (Barnett et al., 2005). Significant work has been undertaken to correct for the errors introduced by this (Gille et al., 2008), and data products including temperature and pressure are now available for scientific use.

The original HIRDLS specification called for the satellite to scan horizontally in azimuth, with a two dimensional mesh of profiles along the orbit track at $500 \mathrm{~km}$ spacing. However, due to the blockage, measurements can only be made at an azimuth of $47^{\circ}$ to the orbital plane of the satellite on the side facing away from the sun. As a result of this, the profiles obtained from the instrument in operation have a much closer horizontal spacing than originally planned, with an alongtrack distance between profiles of $\sim 75-100 \mathrm{~km}$. This allows the detection of much smaller-scale along-track horizontal structures than would have been made under the original mission profile, strongly facilitating gravity wave studies. The vertical projection of the field of view at the limb is $\sim 1 \mathrm{~km}$ in the vertical, and can be oversampled for vertical resolution $<1 \mathrm{~km}$ (Alexander et al., 2008; Gille et al., 2008, 2010). Figure 3 of Alexander and Barnet (2007) once again illustrates this footprint. Around 5600 vertical profiles are measured per day.

Due to the orbital path of the satellite and the effects of the obscuration, the greatest density of profiles are around the northern and southern turnarounds at $\sim 80^{\circ} \mathrm{N}$ and $\sim 65^{\circ} \mathrm{S}$. The orbital path of Aura remains the same throughout the year. After revisions to the scanning pattern to minimise the effects of the blockage, HIRDLS began collecting data on the 21 st of January 2005 (Gille et al., 2010), with some intermittency in the data coverage for a few weeks after this. This study uses HIRDLS V005 data for the period 2006-2007: this is the most current version available. Temperature values have a precision of $\sim 0.5 \mathrm{~K}$ over the height range under consideration.

\subsection{SABER}

The Sounding of the Atmosphere using Broadband Emission Radiometry (SABER) mission is one of four instruments on NASA's TIMED satellite, intended primarily to measure and characterise the mesosphere and lower thermosphere on a global scale. SABER is a 10-channel limb-sounding infrared radiometer, which provides $\sim 2200$ vertical profiles per day with a vertical resolution, again based on the projection of the field of view, of approximately $2 \mathrm{~km}$ (Mertens et al., 2009) and an along-track profile spacing of $\sim 370 \mathrm{~km}$.

SABER's scanning routine incorporates the TIMED spacecraft's yaw cycle, with the coverage region shifting north and south every sixty days to cover the poles alternately. Accordingly, while the coverage of the instrument in the tropics and at midlatitudes remains constant throughout the year, high northerly and southerly latitudes are only covered for 60 in every 120 days, in a 60-days on, 60-days off cycle, with coverage in the the "off" hemisphere extending to $54^{\circ}$ and in the "on" hemisphere to $87^{\circ}$. Kinetic temperature profiles cover the altitude range from $\sim 15 \mathrm{~km}-\sim 120 \mathrm{~km}$ (Mertens et al., 2009; Wrasse et al., 2008). This study uses SABER version 1.07 temperature data for 2006-2007, with a precision of $\sim 0.8 \mathrm{~K}$ (Remsberg et al., 2008).

\section{Profile colocation}

In this study, two-instrument pairs of profiles are directly compared, with stringent criteria applied to determine colocation.

\subsection{Colocation criteria}

When analysing for transient features such as gravity waves in data from multiple sources, it is important to consider only closely-spaced profiles. This can be demonstrated by considering the propagation of a midfrequency gravity wave with time.

For midfrequency waves, the dispersion relation

$\hat{\omega}=N\left|\frac{k_{\mathrm{h}}}{m}\right|=N\left|\frac{\lambda_{\mathrm{z}}}{\lambda_{\mathrm{h}}}\right|$,

applies, for intrinsic frequency $\hat{\omega}$, horizontal and vertical wavenumbers $k_{\mathrm{h}}$ and $m$, and horizontal and vertical wavelengths $\lambda_{\mathrm{h}}$ and $\lambda_{\mathrm{z}}$ (Fritts and Alexander, 2003). For midfrequency gravity waves, typically $\lambda_{\mathrm{z}} \sim 10 \mathrm{~km}$, $\lambda_{\mathrm{h}} \sim 200 \mathrm{~km}$, and a typical stratospheric Brunt-Väisälä frequency $N \sim 0.02 \mathrm{~s}^{-1}$, we estimate an intrinsic frequency $\hat{\omega} \sim 1 \times 10^{-3}$. 

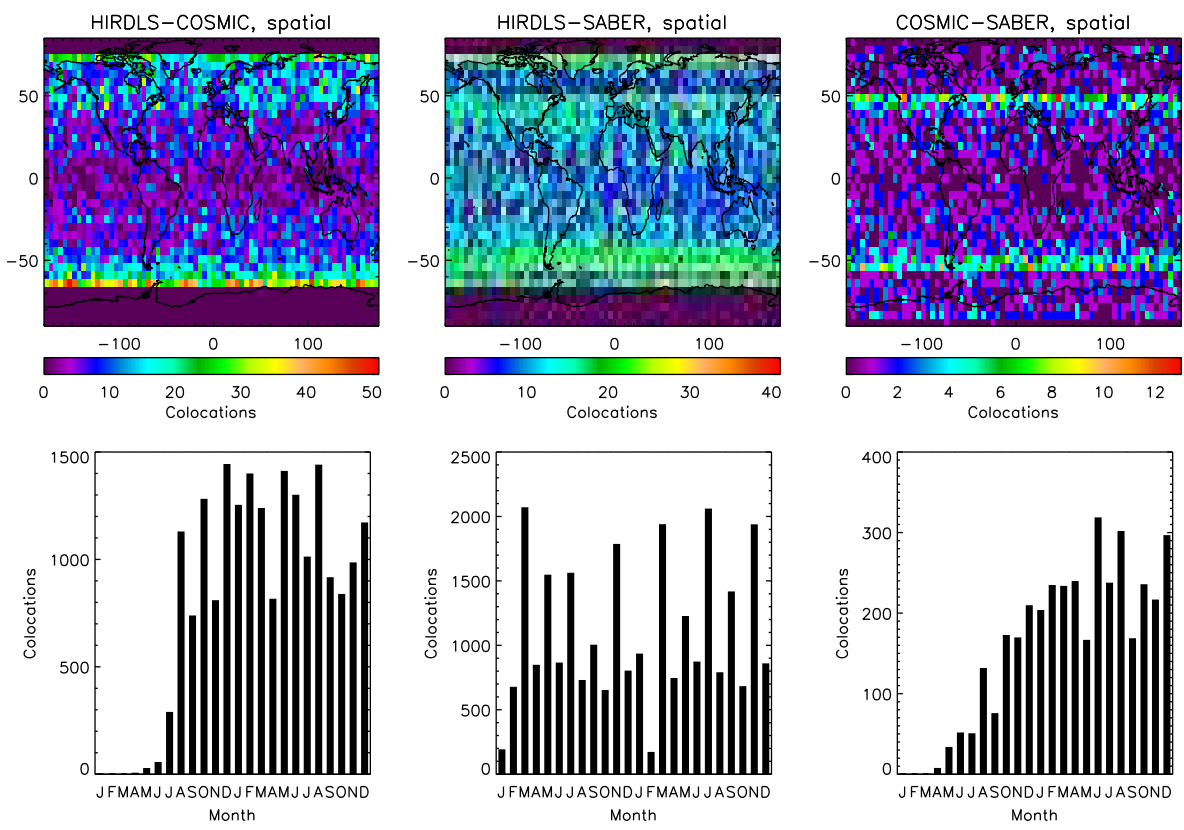

Fig. 1. Details of the temporal and spatial distributions of profile-pairs between each pair of instruments (from left: HIRDLS-COSMIC, HIRDLS-SABER, COSMIC-SABER). In each case, the upper plot shows the geographic distribution of pairs, and the lower plot the temporal distribution.

The group velocity of a wave packet in a background flow of velocity $\overline{u_{\mathrm{h}}}$ is

$\left[c_{\mathrm{g}, \mathrm{h}}, c_{\mathrm{g}, \mathrm{z}}\right]=\left(\overline{u_{\mathrm{h}}}, 0\right)+\left(\frac{\hat{\omega}}{k_{\mathrm{h}}}, \frac{-\hat{\omega}}{m}\right)$,

where $c_{\mathrm{g}, \mathrm{h}}$ is the horizontal group velocity and $c_{\mathrm{g}, \mathrm{z}}$ the vertical. Waves of this type will hence have a horizontal group velocity $c_{\mathrm{g}, \mathrm{h}} \sim 32 \mathrm{~ms}^{-1}$ and a vertical group velocity $c_{\mathrm{g}, \mathrm{z}} \sim 1.4 \mathrm{~ms}^{-1}$. If we consider a time period of a quarter of an hour ( $900 \mathrm{~s}$ ) then, in the absence of a background wind, waves travelling with this group velocity will propagate $1.4 \mathrm{~km}$ vertically, well within the height range covered by our three instruments. We have hence adopted this temporal separation criterion of $900 \mathrm{~s}$.

In the same time, our waves will propagate around $28 \mathrm{~km}$ in the horizontal. This horizontal distance is substantially smaller than the horizontal wavelength of our theoretical midfrequency waves and the atmospheric path length our three instruments average over, both $\sim 180-200 \mathrm{~km}$. To allow for these properties, we shall make profiles lying within $180 \mathrm{~km}$ our spatial colocation requirement.

The three instruments considered are all capable of carrying out stratospheric temperature measurements, but the height range covered by their scanning capabilities differs. Table 1 illustrates these. Based upon these ranges, we observe that the three instruments all cover the range from 100$8 \mathrm{hPa}$, equating approximately to a height range from 16$34 \mathrm{~km}$. However, in the tropics, the tropopause may potentially be as high as $20 \mathrm{~km}$ and give a kink in our smoothed
Table 1. Data specifications for each instrument.

\begin{tabular}{llll}
\hline & COSMIC & HIRDLS & SABER \\
\hline Bottom & $800 \mathrm{hPa}$ & $400 \mathrm{hPa}$ & $135 \mathrm{hPa}$ \\
Top & $7 \mathrm{hPa}$ & $0.4 \mathrm{hPa}$ & $0.0003 \mathrm{hPa}$ \\
Precision & $0.5 \mathrm{~K}$ & $0.5 \mathrm{~K}$ & $0.8 \mathrm{~K}$ \\
Vertical Res. & $\sim 1 \mathrm{~km}$ & $1 \mathrm{~km}$ & $2 \mathrm{~km}$ \\
\hline
\end{tabular}

profiles; whilst this would be seen at the same height in all three instruments, their differing resolutions may lead to the measured location not being observed in quite the same place in colocated measurements. Additionally, this kink may lead to an apparent enhancement in GW activity at the tropopause region (Schmidt et al., 2008). Consequently, a lower altitude bound of $60 \mathrm{hPa}(\sim 20 \mathrm{~km})$ will be used. This range, from $60 \mathrm{hPa}$ to $8 \mathrm{hPa}$, is approximately $10 \times$ the vertical range we would expect a midfrequency gravity wave to propagate vertically in the maximum time between our paired measurements.

\subsection{Number and distribution of colocations}

Figure 1 illustrates the distribution of colocations between the instruments both spatially and temporally over the twoyear period 2006 and 2007. It should be noted that, due to the limitations of the current study and the sampling of colocations, the distributions shown in this figure do not necessarily 
reflect the global distributions of gravity waves observed in previous studies. We shall consider each of these instrument pairs individually.

\subsubsection{HIRDLS-COSMIC}

The left column of Fig. 1 illustrates colocations between HIRDLS and COSMIC. Due to the broad spatial distribution of the profiles produced by both instruments, the geographic profile distribution is generally good across the whole globe and is clearly representative of the input data; the distribution is peaked at the northern and southern turnaround latitudes for HIRDLS, and there are fewer coincidences at tropical latitudes. A large number of colocations are recorded between the two instruments, and these are fairly evenly distributed across the overlapping time period of the two datasets, ramping up initially over a period of months during the commissioning of the COSMIC constellation before reaching a level of $800-1400$ coincidences per month. The total number of coincidences between the two instruments over the period is 19973, of which 19553 provide data over the full height range under consideration and consequently are used in our analysis.

\subsubsection{HIRDLS-SABER}

The centre column of Fig. 1 illustrates HIRDLS-SABER coincidences over the period 2006-2007. As with the HIRDLSCOSMIC coincidences, these are evenly distributed across most latitudes. Although both instruments follow a regular sun-synchronous orbit, they have frequently-intersecting orbits which precess relative to each other. This precession leads to colocations occuring over the full range of latitudes covered by HIRDLS. There is an alternating two-month pattern to the number of coincidences per month; as HIRDLS has good coverage at high northern latitudes but not high southern latitudes, this corresponds directly to the SABER yaw cycle discussed above. 27801 coincidences occur between the instruments, of which 26824 provide data over the full height range for our analysis.

\subsubsection{COSMIC-SABER}

Finally, the right column of Fig. 1 shows COSMIC-SABER coincidences. Once again, the number of colocations ramps up as the COSMIC constellation enters formation, reaching a rate of over 200 coincidences per month. The matches are distributed globally, but with clear peaks at $60^{\circ} \mathrm{N}$ and $60^{\circ} \mathrm{S}$ due to the yaw pattern of TIMED shifting the satellite turnaround to these latitudes at different times of year. 3950 colocations occur between COSMIC and SABER, of which 3871 are suitable for analysis.

\section{Correlation/standard deviation analysis}

\subsection{Methodology}

We shall initially consider our data in terms of the correlation coefficient and standard deviation of colocated profile pairs about their respective smoothed means. In each case, we use the correlation coefficient as a measure of the similarity of the two colocated profiles, and the standard deviation as a proxy to the magnitude of fine structure in the profile. This methodology has previously been used to compare HIRDLS and COSMIC profiles by Barnett et al. (2008).

Initially, each profile is interpolated onto a $200 \mathrm{~m}$ altitude scale, to provide a common scale across all three instruments. The profiles are then convolved with a $7 \mathrm{~km}$ cosine half-bell filter, effectively filtering out any features greater than $14 \mathrm{~km}$ in vertical extent. The new profiles are subtracted from the original (post-interpolation) profiles to produce perturbation profiles; this process acts as a high-pass filter on the data. The high resolution is used in order to represent the half-bell as smoothly as possible. The height region we wish to consider is then extracted.

Figure 2 illustrates this process with a colocated HIRDLSCOSMIC profile pair. From left to right, the first panel shows the original profiles, the second the data interpolated onto the $200 \mathrm{~m}$ scale with the half-bell-smoothed profiles overplotted with dashed lines, the third the perturbation profiles remaining after the half-bell smoothing, and the fourth the height region under investigation extracted. In each case, the coloured lines at the right indicate the height levels under consideration at that stage of the analysis, with orange indicating HIRDLS levels and green COSMIC levels. The standard deviations of the perturbation profiles about their means are then computed, and the profiles are correlated using the linear Pearson correlation coefficient.

This analysis is limited in several ways. Firstly, the interpolation of the datasets to new vertical scales inherently alters the structure of the data. It is necessary for the data to be on the same vertical scale for the correlation and for the standard deviations to be fully comparable, but in performing this step we are adding structural information to the data which was not originally present. Since all the datasets are already oversampled to some degree, however, this step is less of a problem than it could be.

Secondly, and more importantly, this analysis does not tell us what the fine structure under consideration actually is. All that is being considered is the variability of the small-scale vertical structure profiles and their similarity to colocated data: the analysis cannot tell us whether what we are seeing arises due to gravity-wave type features or due to other smallscale atmospheric features such as fine temperature layers. Accordingly, it can only provide us with useful information when considered alongside other analyses.

Third, the highly simplistic smoothing method used to remove the large-scale background does not allow for any 

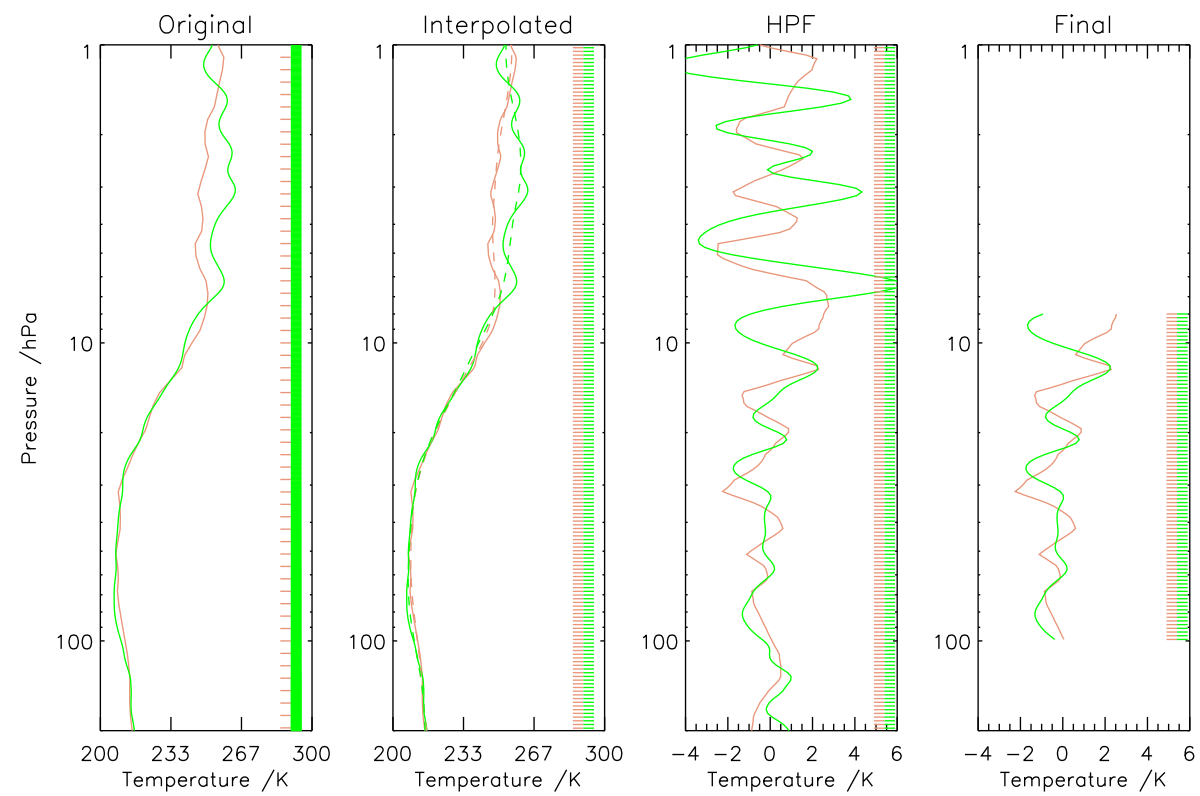

Fig. 2. Illustration of the data preparation applied for our correlation/standard deviation analyses, showing a comparison between a HIRDLS (orange) and COSMIC (green) profile. From left to right, the first panel shows the original profiles, the second the data interpolated onto the $200 \mathrm{~m}$ scale with the half-bell-smoothed profiles overplotted with dashed lines, the third the perturbation profiles remaining after the half-bell smoothing, and the fourth the height region under investigation extracted. In each case, the coloured lines at the right indicate the height levels under consideration at that stage of the analysis, with orange indicating HIRDLS levels and green COSMIC levels. Note that, in this example, the COSMIC profile shown continues to an altitude above $1 \mathrm{hPa}$, whilst in practice most do not.

large-scale planetary wave or, in the tropics, Kelvin wave features which have short vertical wavelength. Consequently, amplitudes and correlations will be high-biased in places where these signals are larger than those due to smaller-scale waves. Indeed, evidence of this is observed in Fig. 6, where larger-amplitude features are shown to be biased towards the tropics.

Finally, it does not take into account the variation between the two profiles due to their time and distance separation and to their different viewing angles. This difference is hopefully minimised by our small time and distance separation criteria, but will lead to differences, particularly in the correlation coefficients between the profile-pairs. In particular, a significant phase difference between the two profiles would result in a low correlation coefficient even if the wave signal detected was identical. This is a particular problem for waves with short horizontal wavelength, where the wave may have several phase cycles between the two measurements.

\subsection{Comparisons}

We shall now discuss the results of the above analysis method as applied to profiles colocated between each pair of instruments (Fig. 3). In each case, we shall consider the number density of correlations and the mean correlation at a given standard deviation for each instrument.

These analyses allow us to consider the correspondence between the correlation quality and the degree of fine structure in the signal. Generalising broadly, we would expect gravity wave signals to be of greater amplitude than background noise, and accordingly would expect profiles with larger amplitudes, and hence larger standard deviations, to be made up to a greater degree of signal rather than noise. Hence, we would expect larger-standard-deviation profiles to be better correlated.

\subsubsection{HIRDLS-COSMIC}

The first row of Fig. 3 illustrates the results of our analysis for colocated HIRDLS and COSMIC profiles.

Considering first the number density plot (left panel) we observe a cluster of high number densities at low standard deviations in both instruments, corresponding to a region made up of uncorrelated profiles (right panel). This is consistent with low-structure noise profiles: profiles with low standard deviations are more likely to represent low-level noise rather than meaningful activity, and consequently would be expected to correlate more weakly. The standard deviations of this cluster are approximately equal to or less than the temperature precision levels of the two instruments, suggesting that the detailed structure of these profiles is close to the noise limits of the two instruments. We also see a bias towards slightly larger standard deviations in the COSMIC data, with the distribution as a whole being skewed towards higher COSMIC values. This is consistent with the results shown in Fig. 4 of Barnett et al. (2008), despite the 

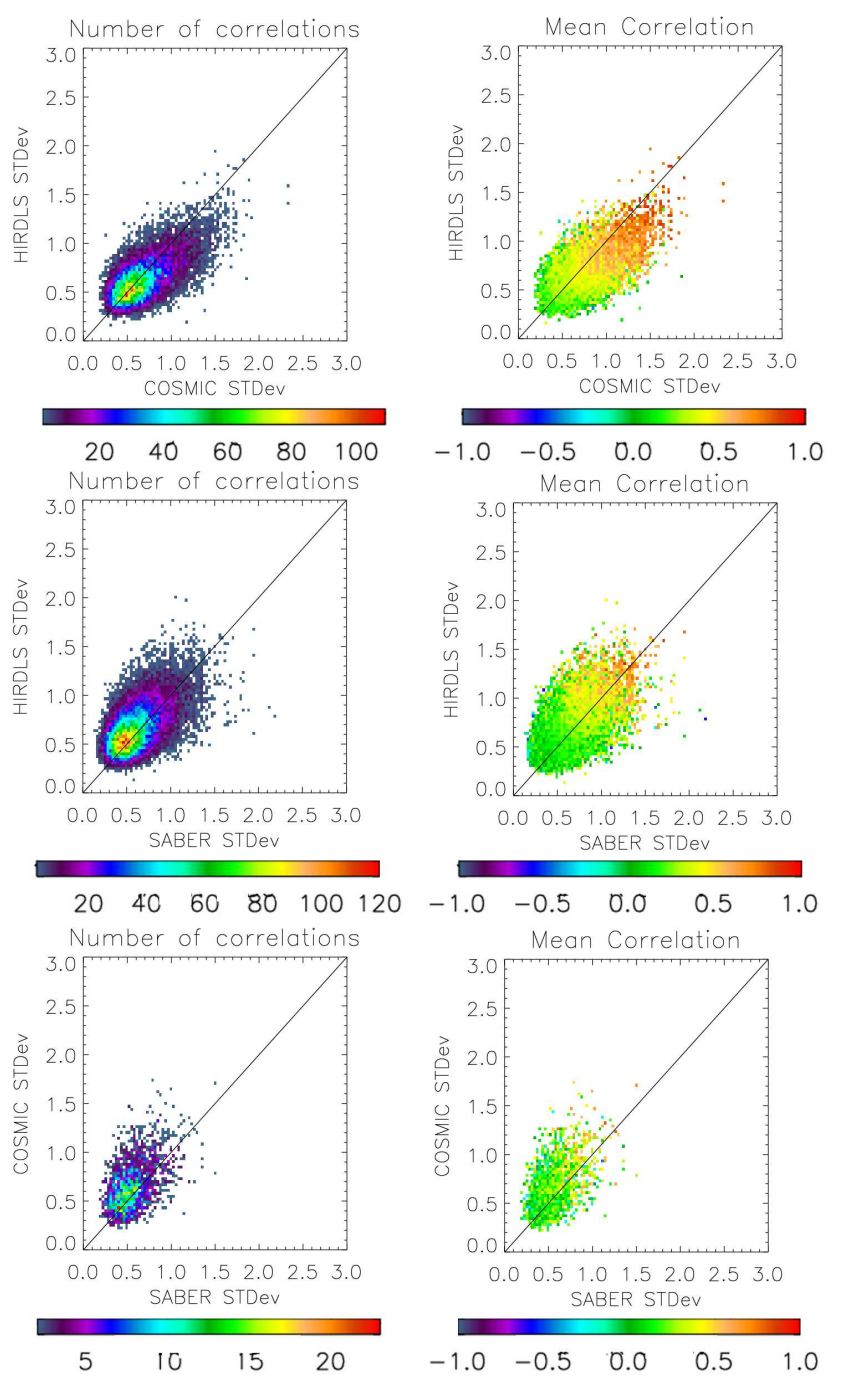

Fig. 3. Correlation-standard deviation analysis output for (top panel) HIRDLS-COSMIC, (middle panel) HIRDLS-SABER and (bottom panel) COSMIC-SABER profile pairs. The left-hand plot shows the data as a scatter of the standard deviations of the two instrument profiles, and the right the same plot but with the correlation values between the two instruments shown. In the left figure, the colour scale represents the number of points in each bin, whilst in the right figure the colour represents the mean correlation for profile-pairs in that bin.

significantly different colocation criteria and different height sampling used. This is indicative of differing resolutions for the two instruments and may be a tail of small-scale fine structure which HIRDLS cannot resolve.

\subsubsection{HIRDLS-SABER}

We next consider the second row of Fig. 3, the comparison between HIRDLS and SABER correlation/standard deviation results. We once again see a pattern of increasing correlation with increasing standard deviation along the 1:1 correspondence line, and a large cluster of low-correlation profiles at low standard deviations. The best-correlating profiles are once again those with the highest standard deviations in both instruments, again suggesting that the profiles with most variability, that is to say the ones with large-amplitude structure rather than low-amplitude noise are the closest in agreement between the two instruments.

\subsubsection{COSMIC-SABER}

Finally, the third row of Fig. 3 illustrates the results for COSMIC-SABER colocations. We again see a pattern of better-correlated high-standard deviation profiles with a large cluster at low standard deviations of uncorrelated profiles. There is a skew towards higher standard deviations in the COSMIC results, consistent with the expected higher vertical resolution of COSMIC.

\subsection{Conclusions}

Whilst the correlation/standard deviation analyses discussed here cannot show unambiguously whether we are correctly detecting gravity wave signals as opposed to atmospheric layering or other similar phenomena, they do show that the three instruments reproduce each others' small-scale structure well, and accordingly could be expected to have similar gravity wave structure. Deviations from agreement between the datasets are generally focused at low standard-deviations, consistent with unstructured noise.

\section{Stockwell transform analysis}

\subsection{Methodology}

Several recent studies, such as those of Alexander et al. (2008, 2009) and Wright et al. (2010), have used the S-Transform (ST, Stockwell et al., 1996), an extension of the continuous wavelet transform based on a scaling movable Gaussian window which provides a time-frequency (or, in the cases of atmospheric profile analysis, height-frequency) representation, to examine satellite temperature profiles for evidence of gravity wave signals. Accordingly, we shall apply this method to compare our profiles for use in gravity wave detection.

Since we are considering only individual profiles, we cannot apply the cospectral analysis used in, e.g. Alexander et al. (2008) and Wright et al. (2010) to analyse the horizontal wavelength of and momentum flux carried by gravity wave signals, and to select only for signals present across multiple profiles. Instead, we consider only the vertical wavelengths and temperature perturbations detected in single STransformed profiles; this yields an estimate of the activity in the individual profile rather than that present across both profiles of an adjacent pair, and hence emphasises significantly more spatially-localised gravity waves. The purpose of this 


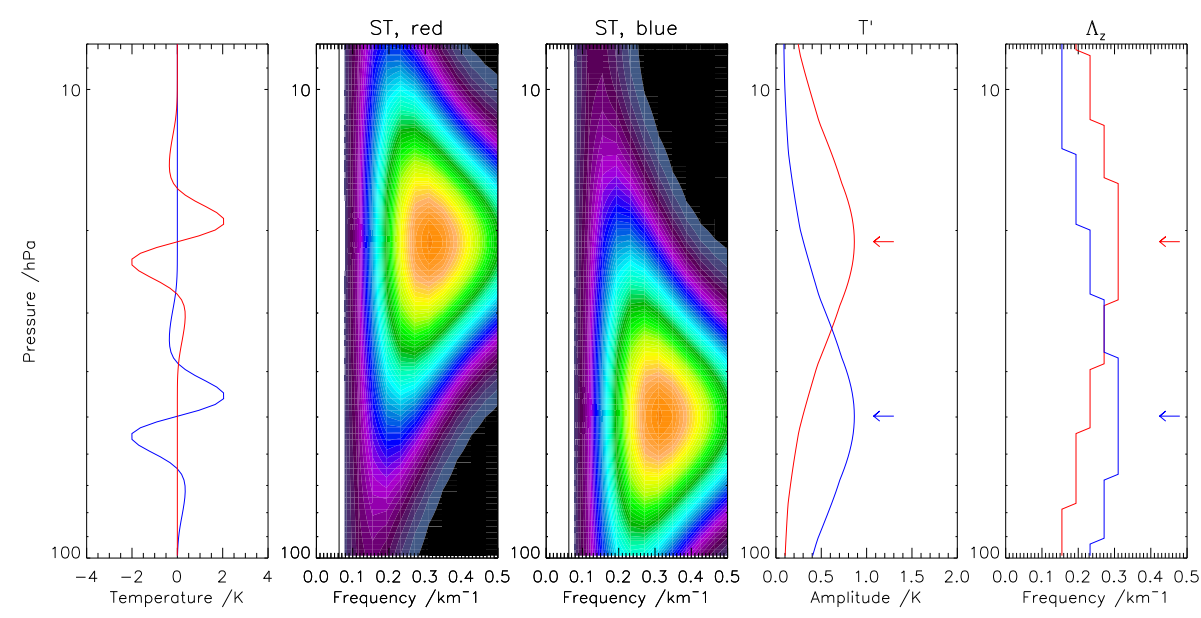

Fig. 4. Sample comparison between S-Transform results for two ideal wave packets, both of the same amplitude and wavelength but but one at low altitude (blue) and the other at high altitude (red). From the left, the first column illustrates two profiles, the second and third the S-Transforms of the two profiles, the fourth the computed peak temperature perturbations as a function of height, and the final column the computed vertical frequencies for these temperature perturbations. The arrows on the two output plots indicate the temperature maxima selected from the two profiles and their associated vertical wavelengths. Our analysis would identify these waves as equal in temperature pertubation and wavelength despite their different altitude, under the assumption that the time separation accounted for their different altitudes.

is to assess how well the three instruments estimate the vertical wavelengths and temperature perturbations of the profiles under consideration relative to each other.

Figure 4 illustrates the method used to carry out this analysis. Two ideal waves are shown, but the same approach is used for the real data, with colocated profile pairs filtered using the half-cosine bell approach outlined above used as the input. The profiles are individually S-Transformed (figures in second and third columns from left). We then extract the peak temperature perturbation at each height level in the profile (second panel from right), and the vertical wavelength of this signal (right panel). We repeat this analysis for each colocated profile-pair.

Figure 5 shows the results of these analyses on a wholedataset scale. For each row, the left-hand figure shows, on each axis, the peak temperature perturbation in each individual profile, as indicated by the arrows on Fig. 4, with results binned on a $0.075 \mathrm{~K}$ by $0.075 \mathrm{~K}$ scale. These points are not necessarily at the same height level; they indicate only the individual peaks in the profiles. This selection makes sense if considered in terms of an individual gravity wavepacket propagating between the two measurements: in the time between the two profiles being measured, the wavepacket will propagate some distance vertically (and also horizontally), and accordingly a measurement at the same height would fail to detect the corresponding signal. By selecting in this way, any wave packets with temperature amplitude significantly above the local mean will accordingly be associated with each other across the profile pair. The right-hand plots indicate the measured vertical frequency of this temperature maximum in each profile, binned on a $0.04 \mathrm{~km}^{-1}$ by
$0.04 \mathrm{~km}^{-1}$; applying the same logic, this should show a good match for high-amplitude wavepackets separated in height but representing the same wave signal. On both of these plots, the solid diagonal line indicates a 1:1 correspondence between the two datasets.

To perform this analysis, we have considered only the subset of profiles from the previous analysis which showed indications of significant activity. Specifically, only profilepairs where the standard deviation in each profile is greater than $0.8 \mathrm{~K}$ have been considered. This criterion, rather than one based on their correlation coefficients as previously computed, is used to allow for variation of wavepacket height between the two measurements: to take an extreme case, a perfectly-resolved gravity wavepacket with no background noise which was reproduced perfectly between the two measurements except for a large height change would return a correlation coefficient of zero, but identical standard deviations, from our previous analysis. This serves to remove the large peak of low-amplitude noise present in our previous analysis from the data, hopefully providing a better test of our analysis method.

Figure 6 shows the distribution of profiles remaining after this selection has taken place. This distribution, weighted towards tropical regions, is representative spatially of the gravity wave climatology as observed by detection methods focusing upon short vertical wavelength waves (Alexander and Barnet, 2007). All three instruments retain their global coverage; the temporal distribution of the pairings remains broadly similar, but with a much stronger reduction in alternate months of HIRDLS-SABER colocations due to the TIMED yaw cycle. There is also a stronger emphasis on 

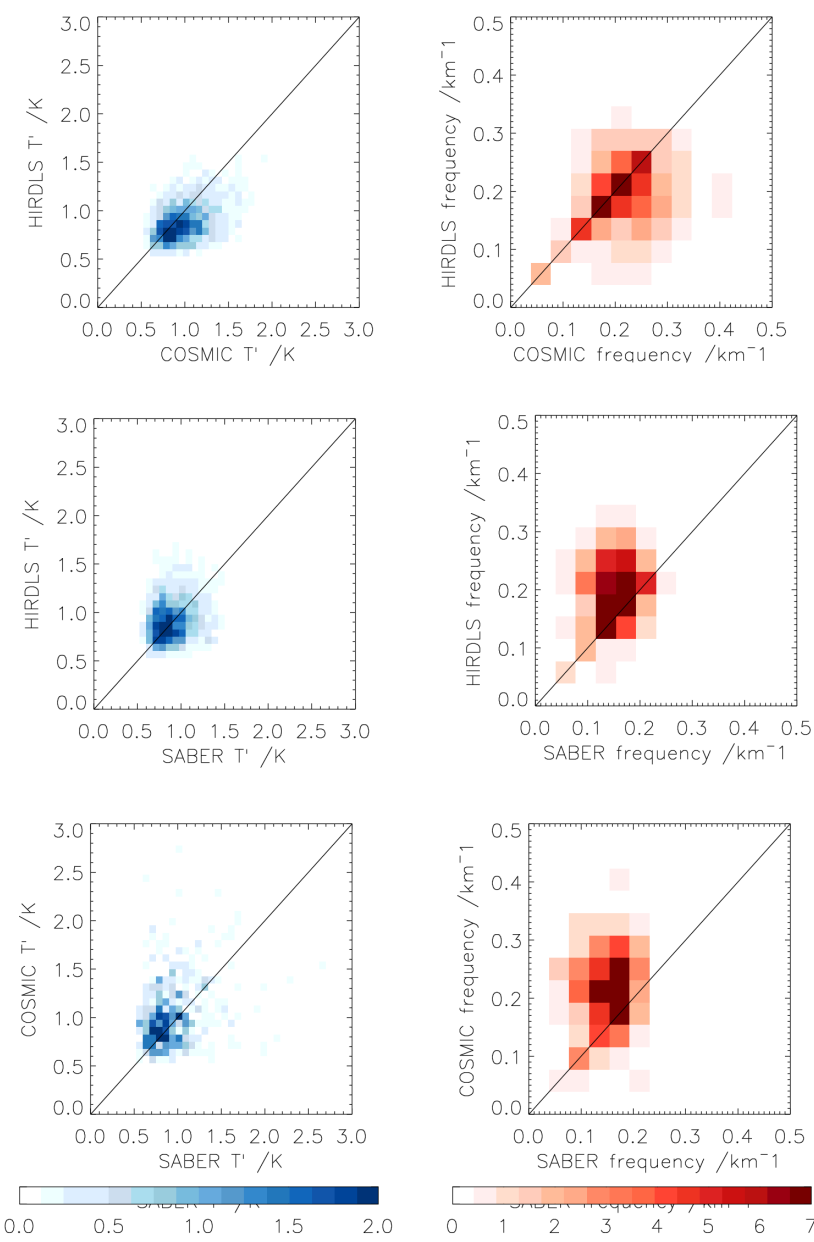

Fig. 5. ST analysis output for (top panel) HIRDLS-COSMIC, (middle panel) HIRDLS-SABER and (bottom panel) COSMIC-SABER profile pairs. The left plot shows a density plot of the peak temperature perturbation in each paired profiles, and the right a density plot of the vertical frequencies of these temperature perturbation peaks. Scales are as a percentage of the total number of analysed profilepairs in each case.

midlatitudinal and tropical results than with the unfiltered data, with a much smaller proportion of results at Arctic and Antarctic latitudes.

It should be noted that, due to the decrease of atmospheric density with height, we would expect the magnitude of temperature perturbations due to gravity waves to increase with increasing altitude; for example, Alexander et al. (2008) show perturbations growing by $\sim 50-60 \%$ over this range. Such an increase is also observed in our results (not shown). Accordingly, basing our analysis on the largest temperature perturbation present in each profile will inevitably bias our results towards signals at higher altitudes. Nevertheless, this process still compares like-with-like across each profile-pair, and hence provides useful comparative results. In particular, for a gravity wavepacket of significantly greater amplitude than the mean we should still resolve the same feature at all heights.

\subsection{Comparisons}

\subsubsection{HIRDLS-COSMIC}

The top row of Fig. 5 illustrates the results obtained from the ST comparison of HIRDLS and COSMIC. Considering first the temperature analysis in the left-hand figure, we observe the same bias in the temperature perturbations measured towards COSMIC as in the first row of Fig. 3: the profilepeak temperature perturbation scatterplot shows a marked bias in favour of higher values in COSMIC, with the distribution skewing away from the 1:1 correspondence line. The scatterplot for frequency shows good agreement, with the bulk of pairings tightly distributed about the 1:1 correspondence line but with a slight bias towards higher frequencies (shorter wavelengths) being observed by COSMIC: in particular, no results are observed for HIRDLS with a frequency greater than $0.35 \mathrm{~km}^{-1}$, whereas COSMIC results continue to be observed up to $0.45 \mathrm{~km}^{-1}$, albeit in very small numbers. These results are consistent with COSMIC potentially having a slightly higher vertical resolution than estimated: some small-scale features which would be fully resolved at the resolution of COSMIC will not be observed by HIRDLS, leading to this result.

\subsubsection{HIRDLS-SABER}

The middle row of Fig. 5 shows results for the ST comparison of HIRDLS and SABER. The temperature scatterplot shows general agreement, although with a wider spread of values than the previous HIRDLS-COSMIC comparison. The frequency scatterplot shows much less agreement, with the distribution heavily skewed towards lower frequencies (longer wavelengths) in the SABER data, and a cutoff at $0.25 \mathrm{~km}^{-1}$. The bulk of results obtained, however, remain on the 1:1 correspondence line. This is consistent with the coarser vertical resolution of SABER by comparison with HIRDLS and COSMIC: for a wave to be Nyquist sampled by the $2 \mathrm{~km}$ vertical resolution of SABER, the maximum frequency of the wave would be 0.25 cycles per kilometre, in agreement with the results observed, and highlights that SABER cannot be used to detect waves with vertical frequencies greater than this. This also explains the poorer fit for temperature perturbations: many of the results obtained will in fact be for a wavelike feature which may be properly observed only by HIRDLS in this pairing.

\subsubsection{COSMIC-SABER}

Finally, we consider COSMIC-SABER pairings (bottom row of Fig. 5). The significantly smaller number of colocations as compared to the other two instrument pairings makes this analysis weaker, but similar results to the 

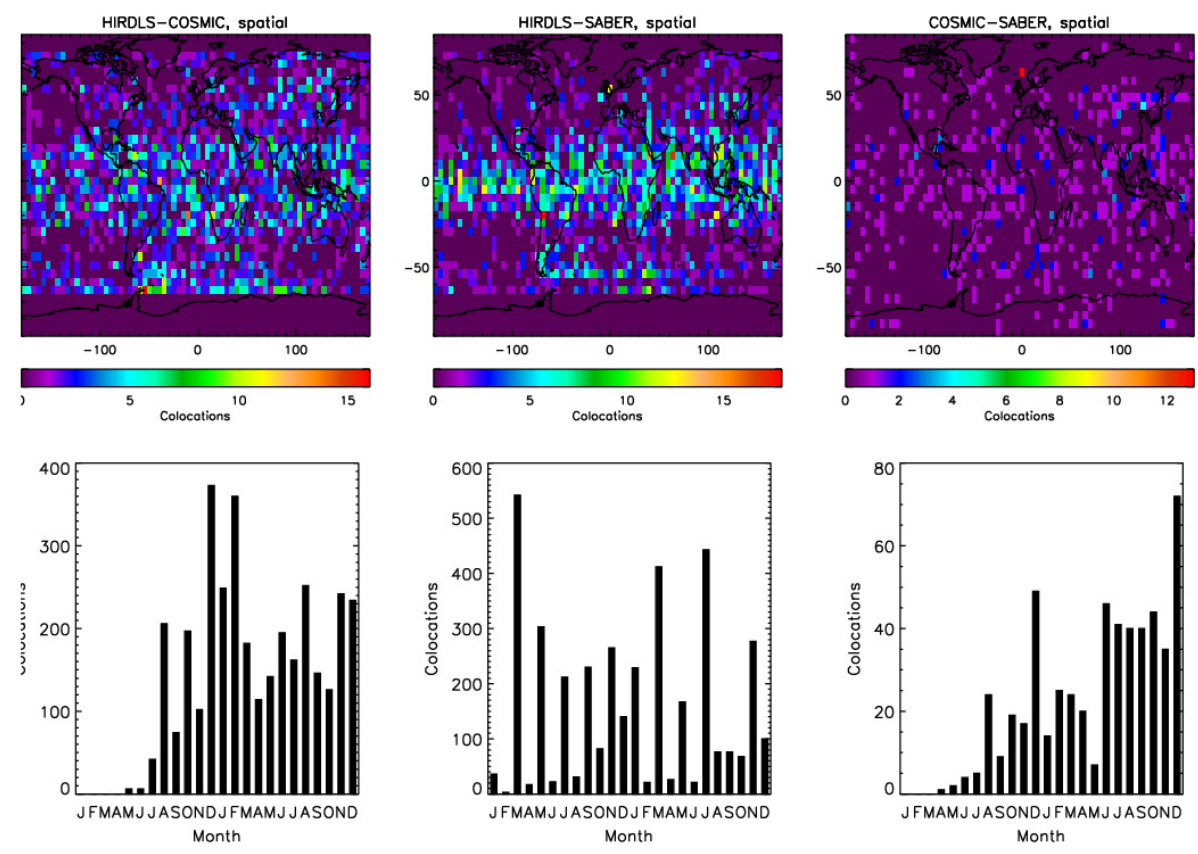

Fig. 6. Details of the temporal and spatial distributions of profile-pairs between each pair of instruments (from left: HIRDLS-COSMIC, HIRDLS-SABER, COSMIC-SABER) after removing low-standard deviation profiles.

HIRDLS-SABER comparisons are obtained, with a sharp skew towards smaller-scale structure in COSMIC and results much more divergent than in the HIRDLS-COSMIC and HIRDLS-SABER comparisons.

\subsection{Conclusions}

From our S-Transform analyses, we conclude that the three results reproduce each others' results reasonably to within their resolution limits. Temperature perturbation comparisons for each instrument pair hew generally to the 1:1 correspondence line, with slight skews towards larger perturbations observed for pairs involving COSMIC results, but with fairly broad distributions. Vertical wavelength comparisons are also generally distributed about the 1:1 correspondence line in the bulk of cases, with significant deviations due to the lower resolution of SABER as compared to the other two instruments. A slight deviation in favour of COSMIC having a slightly higher vertical resolution than HIRDLS is observed, but the distribution is again fairly wide.

\section{Discussion and conclusions}

Based upon our analyses, we conclude that the three instruments reproduce each others' results recognisably, but not exactly: while the three datasets reproduce each other fairly closely when considered in terms of their standard deviations and correlations at large amplitudes (Sect. 4.2.1), the more detailed S-transform analyses of these high-amplitude profiles return a broad distribution of results. Whilst more than $50 \%$ of the results lie distributed on or close to the correspondence line in all cases, a significant proportion do not.

The datasets were also analysed for three-way coincidences between HIRDLS, COSMIC and SABER. Only 13 three-way colocations with standard deviations in all three instruments greater than $0.8 \mathrm{~K}$ were found, too small a number to draw meaningful conclusions from, and accordingly these results have been omitted. Comparisons were also undertaken between the three instruments shown, the Michelson Interferometer for Passive Atmospheric Sounding (MIPAS, Fischer et al., 2000) and the Solar Occultation for Ice Experiment (SOFIE, Gordley et al., 2009). These two instruments have significantly coarser stratospheric vertical resolutions than SABER, HIRDLS, and COSMIC $(\sim 3 \mathrm{~km}$ for MIPAS, $\sim 3.5 \mathrm{~km}$ for SOFIE), and accordingly gave significantly poorer comparative results for both analyses, with no significant correlations for their standard deviations, temperature perturbations or vertical wavelengths.

Based upon our results, we can proceed to draw some additional conclusions with regards to gravity wave analysis with the three instruments.

The comparatively low vertical resolution of SABER makes it the weakest for this type of analysis, with significant parts of the small-scale frequency spectrum being omitted from the results obtained. SABER does, however, have the longest data record, running continuously from 2002 to the present day, and can study the largest altitude range.

HIRDLS performs better vertically, measuring much higher vertical frequencies, and also has by far the largest 
number of profiles per day, $\sim 5600$, allowing comparatively fine horizontal structure to be detected. This allows the computation of momentum fluxes due to gravity waves comparatively accurately, provided the satellite scan track is aligned at a close angle to the wavevector (Ern et al., 2004).

Finally, COSMIC performs better than either instrument in vertical resolution, with a potential vertical resolution in this height range as good as $700 \mathrm{~m}$, although this is only observed in a very small number of cases. However, the pseudorandom nature of the COSMIC profile distribution combined with the smaller number of profiles per day limit its use in determining momentum fluxes: although this can be done, for example using the method described in Wang and Alexander (2010), it requires considerable averaging over large geographic regions.

Acknowledgements. Portions of the work by the lead author were carried out at the University of Oxford, funded by a DPhil studentship from the UK Natural Environment Research Council (NERC) and under the supervision of J. J. Barnett (deceased). The authors are currently funded by NASA's Aura satellite program under contract NAS5-97046.

The authors would also like to thank Karl Hudnut for help obtaining the COSMIC data, Chris Rocken for advice on the vertical resolution of COSMIC, Steve Massie, Rebecca Batchelor, and two anonymous reviewers for useful revisions to the manuscript, and the whole HIRDLS team for many years of dedicated work.

The National Center for Atmospheric Research is sponsored by the National Science Foundation. Any opinions, findings and conclusions or recommendations expressed in the publication are those of the author(s) and do not necessarily reflect the views of the National Science Foundation.

Edited by: M. Riese

\section{References}

Alexander, M. J. and Barnet, C.: Using Satellite Observations to Constrain Paramerizations of Gravity Wave Effects for Global Models, J. Atmos. Sci., 64, 1652-1665, 2007.

Alexander, M. J., Gille, J., Cavanaugh, C., Coffey, M., Craig, C., Dean, V., Eden, T., Francis, G., Halvorson, C., Hannigan, J., Khosravi, R., Kinneson, D., Lee, H., Massie, S., Nardi, B., and Lambert, A.: Global Estimates of Gravity Wave Momentum Flux from High Resolution Dynamics Limb Sounder (HIRDLS) Observations, J. Geophys. Res., 113, D15S18, doi:10.1029/2007JD008807, 2008.

Alexander, M. J., Eckermann, S. D., Broutman, D., and Ma, J.: Momentum flux estimates for South Georgia Island mountain waves in the stratosphere observed via satellite, Geophys. Res. Lett., 36, L12816, doi:10.1029/2009GL038587, 2009.

Anthes, R. A., Ector, D., Hunt, D. C., Kuo, Y. H., Rocken, C., Schreiner, W. S., Sokolovskiy, S. V., Syndergaard, S., Wee, T. K., Zeng, Z., Bernhardt, P. A., Dymond, K. F., Chen, Y., Liu, H., Manning, K., Randel, W. J., Trenberth, K. E., Cucurull, L., Healy, S. B., Ho, S. P., McCormick, C., Meehan, T. K.,
Thompson, D. C., and Yen, N. L.: The COSMIC/FORMOSAT3 Mission: Early Results, B. Am. Meteorol. Soc., 89, 313-333, doi:10.1175/BAMS-89-3-313, 2008.

Barnett, J. J., Hepplewhite, C. L., Rokke, L., and Gille, J. C.: Mapping the optical obscuration in the NASA Aura HIRDLS instrument, Proc. SPIE, 5883, I1-I10, 2005.

Barnett, J. J., Hepplewhite, C. L., Osprey, S., Gille, J. C., and Khosravi, R.: Cross-validation of HIRDLS and COSMIC radiooccultation retrievals, particularly in relation to fine vertical structure, Proc. SPIE, 7082, 708216, doi:10.1117/12.800702, 2008.

Ern, M., Preusse, P., Alexander, M., and Warner, C. D.: Absolute values of gravity wave momentum flux derived from satellite data, J. Geophys. Res., 109, D20103, doi:10.1029/2004JD004752, 2004.

Fischer, H., Blom, C., Oelhaf, H., Carli, B., Carlotti, M., Delbouille, L., Ehhalt, D., Flaud, J., Isaksen, I., Lopez-Puertas, M., McElroy, C., and Zander, R.: ENVISAT-MIPAS, the Michelson Interferometer for Passive Atmospheric Sounding; An instrument for atmospheric chemistry and Climate Research ENVISAT Mipas An instrument for Atmospheric Chemistry and Climate Research, ESA, 2000.

Fritts, D. C.: Gravity Wave Saturation in the Middle Atmosphere: A Review of Theory and Observations , Rev. Geophys., 22, 275308, 1984.

Fritts, D. C. and Alexander, M. J.: Gravity Wave Dynamics and Effects in the Middle Atmosphere, Rev. Geophys., 41, 1, doi:10.1029/2001RG000106, 2003.

Fritts, D. C. and Luo, Z.: Gravity Wave Excitation by Geostrophic Adjustment of the Jet Stream, Part I: Two-Dimensional Forcing, J. Atmos. Sci., 49, 681-697, 1992.

Gille, J., Barnett, J., Whitney, J., Dials, M., Woodard, D., Rudolf, W., Lambert, A., and Mankin, W.: The High Resolution Dynamics Limb Sounder (HIRDLS) Experiment on Aura, Proc. SPIE, 5152, 162-171, 2003.

Gille, J., Barnett, J., Arter, P., Barker, M., Bernath, P., Boone, C., Cavanaugh, C., Chow, J., Coffey, M., Craft, J., Craig, C., Dials, M., Dean, V., Eden, T., Edwards, D. P., Francis, G., Halvorson, C., Harvey, L., Hepplewhite, C., Khosravi, R., Kinnison, D., Krinsky, C., Lambert, A., Lee, H., Lyjak, L., Loh, J., mankin, W., Massie, S., McInerney, J., Moorhouse, J., Nardi, B., Packman, D., Randall, C., Reburn, J., Rudolf, W., Schwartz, M., Serafin, J., Stone, K., Torpy, B., Walker, K., Waterfall, A., Watkins, R., Whitney, J., Woodard, D., and Young, G.: High Resolution Dynamics Limb Sounder: Experiment overview, recovery and validation of initial temperature data, J. Geophys. Res., 113, D16S43, doi:10.1029/2007JD008824, 2008.

Gille, J., Barnett, J., and The HIRDLS Team: High Resolution Dynamics Limb Sounder, Earth Observing System (EOS) - Data Description and Quality, Version 5, HIRDLS group, 2010.

Gordley, L. L., Hervig, M. E., Fish III, C., Bailey, S., Cook, J., Hansen, S., Shumway, A., Paxton, G., Deaver, L., Marshall, T., Burton, J., Magill, B., Brown, C., Thompson, E., and Kemp, J.: The Solar Occultation For Ice Experiment, J. Atmos. Sol.-Terr. Phy., 71, 300-315, doi:10.1016/j.jastp.2008.07.012, 2009.

Karoly, D. J., Roof, G. L., and Reeder, M. J.: Gravity wave activity association with tropical convection detected in TOGA COARE sounding data, Geophys. Res. Lett., 23, 261-264, 1996. 
Lane, T. P., Reeder, M. J., and Clark, T. L.: Numerical Modelling of Gravity Wave Generation by Deep Tropical Convection, J. Atmos. Sci., 58, 1249-1274, 2001.

Mertens, C., Russell, J., Mlynczak, M., She, C., Schmidlin, F., Goldberg, R., Lopezpuertas, M., Wintersteiner, P., Picard, R., and Winick, J.: Kinetic temperature and carbon dioxide from broadband infrared limb emission measurements taken from the TIMED/SABER instrument, Adv. Space Res., 43, 15-27, doi:10.1016/j.asr.2008.04.017, 2009.

Nappo, C. J.: An Introduction to Atmospheric Gravity Waves, International Geophysics Series, Academic Press, San Diego, USA, 2002.

Remsberg, E. E., Marshall, B. T., Garcia-Comas, M., Krueger, D., Lingenfelser, G. S., Martin-Torres, J., Mlynczak, M. G., Russell, J. M., Smith, A. K., Zhao, Y., Brown, C., Gordley, L. L., Lopez-Gonzalez, M. J., Lopez-Puertas, M., She, C. Y., Taylor, M. J., and Thompson, R. E.: Assessment of the quality of the Version 1.07 temperature-versus-pressure profiles of the middle atmosphere from TIMED/SABER, J. Geophys. Res., 113, D17101, doi:10.1029/2008JD010013, 2008.
Schmidt, T., de la Torre, A., and Wickert, J.: Global gravity wave activity in the tropopause region from CHAMP radio occultation data, Geophys. Res. Lett., 35, L16807, doi:10.1029/2008GL034986, 2008.

Stockwell, R. G., Mansinha, L., and Lowe, R. P.: Localization of the Complex Spectrum: The S Transform, IEEE T. Signal Proc., 44, 998-1001, 1996.

Wang, L. and Alexander, M. J.: Global estimates of gravity wave parameters from GPS radio occultation temperature data, J. Geophys. Res., 115, D21122, doi:10.1029/2010JD013860, 2010.

Wrasse, C., Fechine, J., Takahashi, H., Denardini, C., Wickert, J., Mlynczak, M., Russell, J., and Barbosa, C.: Temperature comparison between CHAMP radio occultation and TIMED/SABER measurements in the lower stratosphere, Adv. Space Res., 41, 1423-1428, doi:10.1016/j.asr.2007.06.073, 2008.

Wright, C. J., Osprey, S. M., Barnett, J. J., Gray, L. J., and Gille, J. C.: High Resolution Dynamics Limb Sounder Measurements of Gravity Wave Activity in the 2006 Arctic Stratosphere, J. Geophys. Res., 115, D02105, doi:10.1029/2009JD011858, 2010. 